\title{
Case \\ Report \\ A Case of Intraprocedural Plaque Protrusion during Carotid Artery Stenting Using the Stent-in-Stent Technique for Carotid Artery Stenosis with Unstable Plaque
}

Hidehiko Taguchi, ${ }^{1,2,3}$ Katsutoshi Takayama, ${ }^{1}$ Hayato Kishida, ${ }^{1}$ Takeshi Wada, ${ }^{1}$ Kaoru Myouchin, ${ }^{3}$ Toshihiro Tanaka, ${ }^{3}$ and Kimihiko Kichikawa ${ }^{3}$

Objective: Carotid artery stenting (CAS) using the stent-in-stent technique was reported to prevent intraprocedural plaque protrusion (PP) in patients with carotid artery stenosis with unstable plaque. We report a case of intraoperative PP after CAS despite the use of stent-in-stent technique.

Case Presentation: A 63-year-old man presented with rapid progression of right carotid artery stenosis with unstable plaque during follow-up and was admitted to undergo CAS. Under local anesthesia with Mo.Ma Ultra and FilterWire EZ protection, CAS was performed using the stent-in-stent technique. The first $8 \mathrm{~mm} \times 29 \mathrm{~mm}$ Carotid Wallstent (CWS) was placed. The second CWS $(6 \mathrm{~mm} \times 22 \mathrm{~mm})$ was placed in a stent-in-stent manner to match the stenotic lesion, and conservative postdilation was performed. Then the third CWS $(6 \mathrm{~mm} \times 22 \mathrm{~mm})$ was added due to the presence of PP on intravascular ultrasonography (IVUS). No postoperative neurological abnormalities were found, and no new high-signal areas were observed on diffusion-weighted MRI the day after surgery. The patient was discharged without postoperative complications. No stroke and restenosis were observed at 3 months after CAS.

Conclusion: PP can occur even with stent-in-stent technique, suggesting the importance of diagnosis by IVUS.

Keywords $>$ CAS, plaque protrusion, stent-in-stent, IVUS

\section{Introduction}

Carotid artery stenting (CAS) is approved for high- and standard-risk patients for carotid endarterectomy (CEA). ${ }^{1,2)}$

${ }^{1}$ Department of Interventional Neuroradiology and Radiology, Koseikai Takai Hospital, Tenri, Nara, Japan

${ }^{2}$ Department of Radiology, Higashiosaka City Medical Center, Higashiosaka, Osaka, Japan

${ }^{3}$ Department of Radiology, Nara Medical University, Kashihara, Nara, Japan

Received: December 4, 2020; Accepted: March 14, 2021 Corresponding author: Hidehiko Taguchi. Department of Radiology, Higashiosaka City Medical Center, 3-4-5, Nishiiwata, Higashiosaka, Osaka 578-8588, Japan

Email: hidehiko0912@gmail.com

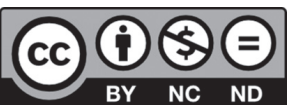

This work is licensed under a Creative Commons Attribution-NonCommercialNoDerivatives International License.

(C)2021 The Japanese Society for Neuroendovascular Therapy
However, cerebral infarction as a perioperative complication related to CAS is an issue, and previous studies reported that risk factors for cerebral infarction included protection devices, operator's skill, patient age, plaque properties, stent design, and statin ${ }^{3)}$ use. Kotsugi et al. ${ }^{4)}$ reported that plaque protrusion (PP), as a new risk factor, was strongly associated with cerebral infarction. To prevent perioperative cerebral infarction, it may be necessary to select a procedure and devices that do not induce PP. Furthermore, Kotsugi et al.4) found that unstable plaque and open-cell stent use were predictive factors for PP. As a method to prevent PP in the case of unstable plaque, Myouchin et al. ${ }^{5)}$ proposed the stent-in-stent technique with closed-cell stents. At our institution, CAS for unstable plaque is usually conducted with two overlapping closed-cell stents to prevent PP according to this article. In this study, we report a patient in whom the stent-in-stent technique with closedcell stents was performed to treat carotid artery stenosis with unstable plaque, but PP occurred during CAS. 


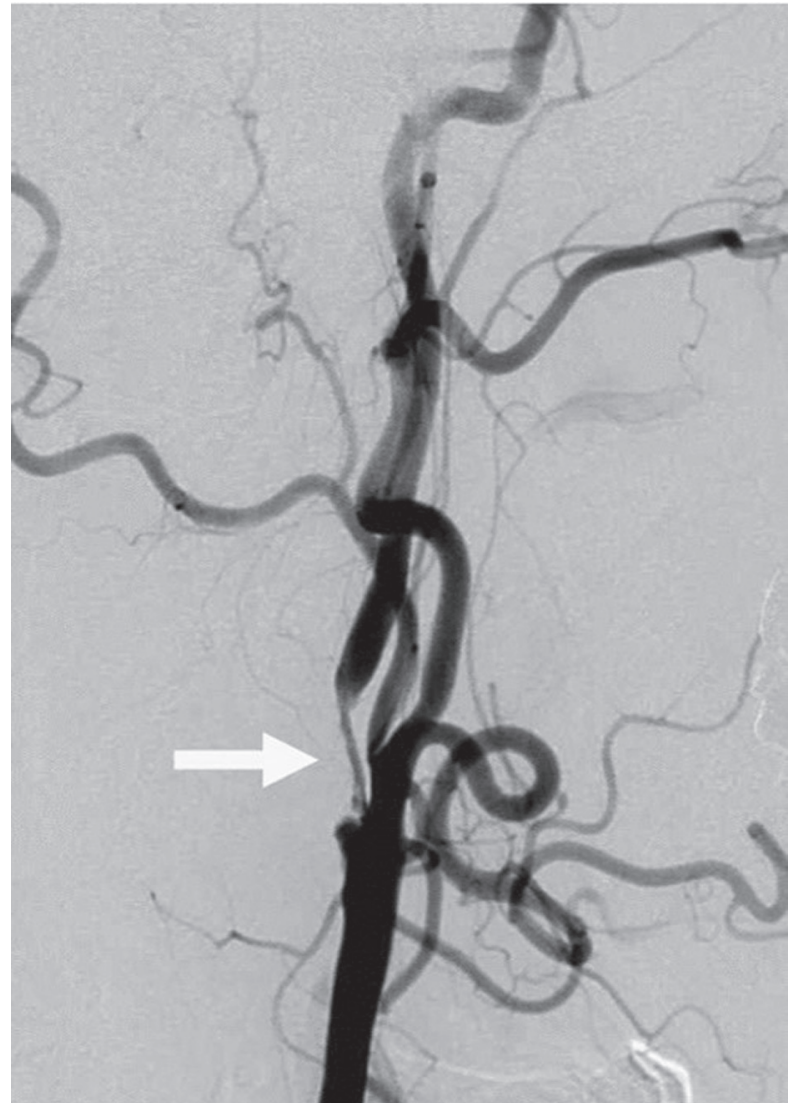

Fig. 1 Right common carotid angiography before CAS (lateral view) showing $90 \%$ long stenosis (arrow) at the origin of the right ICA. CAS: carotid artery stenting; ICA: internal carotid artery

\section{Case Presentation}

Patient: A 63-year-old man.

Chief complaint: Progression of right internal carotid artery (ICA) stenosis.

Present illness: During follow-up of right ICA stenosis, it rapidly progressed from $50 \%$ to $90 \%$ on the North American Symptomatic Carotid Endarterectomy Trial (NASCET) method in 6 months, and the patient was admitted for CAS. Neurological findings on admission: No abnormal findings. Medical history: Clipping of an unruptured anterior communicating artery aneurysm (2018).

Risk factors for arteriosclerosis: Hypertension, dyslipidemia, and previous smoking ( 10 cigarettes per day for 40 years).

\section{Neuroradiological findings Cerebral angiography}

At the origin of the right ICA, NASCET $90 \%$ stenosis measuring approximately $2.5 \mathrm{~cm}$ in length was observed (Fig. 1).

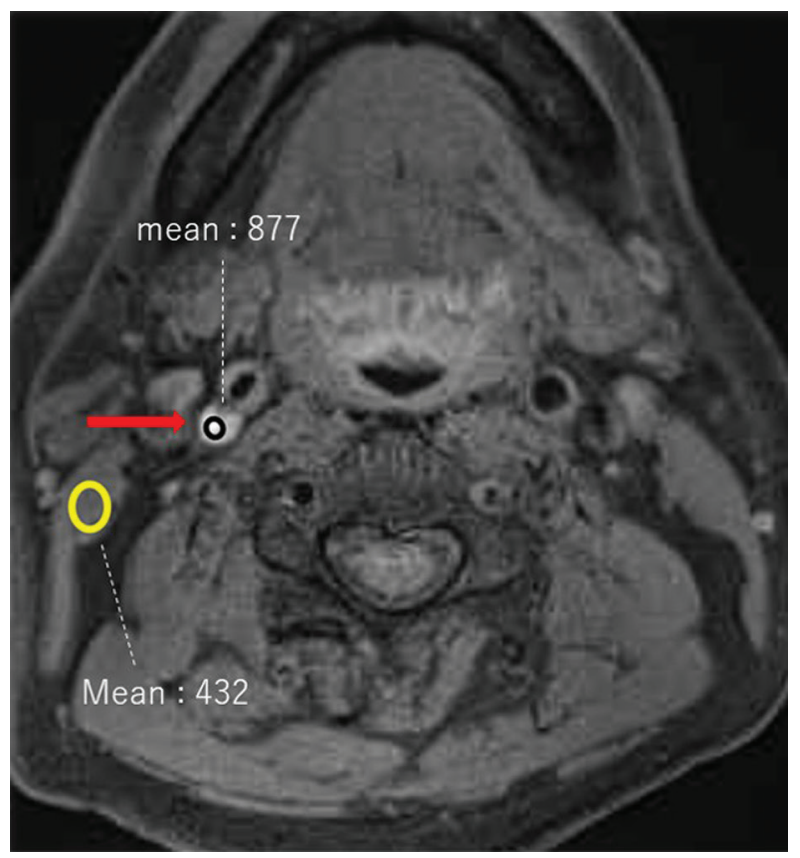

Fig. 2 Preoperative magnetic resonance plaque imaging with T1-weighted black blood method (axial image). A high signal intensity area is evident at the origin of the right ICA (arrow). The black circle shows the ROI at the carotid plaque (mean signal intensity: 877 ) and the yellow circle shows the ROI at muscle (mean signal intensity: 432). The signal intensity ratio is $2: 1$, suggesting the presence of unstable plaque. ICA: internal carotid artery; ROI: region of interest

\section{Magnetic resonance plaque imaging}

Plaque at the site of right ICA stenosis showed markedly high signal intensity in T1-weighted image (axial image) using the black blood method. The sternocleidomastoid muscle and plaque signal intensity ratio was $2: 1$, suggesting unstable plaque (Fig. 2).

\section{Neuroendovascular treatment}

$100 \mathrm{mg}$ of aspirin and $75 \mathrm{mg}$ of clopidogrel were orally administered 2 weeks before CAS. Using a VerifyNow system (Accumetrics, San Diego, CA, USA), we confirmed that the aspirin reaction units and $\mathrm{P} 2 \mathrm{Y} 12$ reaction units were 570 and 77 , respectively, being almost within the effective ranges, 10 days before the procedure (Fig. 3).

Under local anesthesia, an 8-Fr. long sheath was inserted through the right femoral artery. After intraoperative systemic heparinization (activated clotting time $\geq 275$ ), the following two protection devices were inserted: an 8-Fr. Mo.Ma Ultra (Medtronic Vascular, Santa Rosa, CA, USA) and FilterWire EZ (Boston Scientific, Natick, MA, USA). The lesion was crossed with the FilterWire EZ and predilation at the site of stenosis was performed using a $3.0 \mathrm{~mm} \times 40 \mathrm{~mm}$ balloon catheter (Bellona; Medicos 


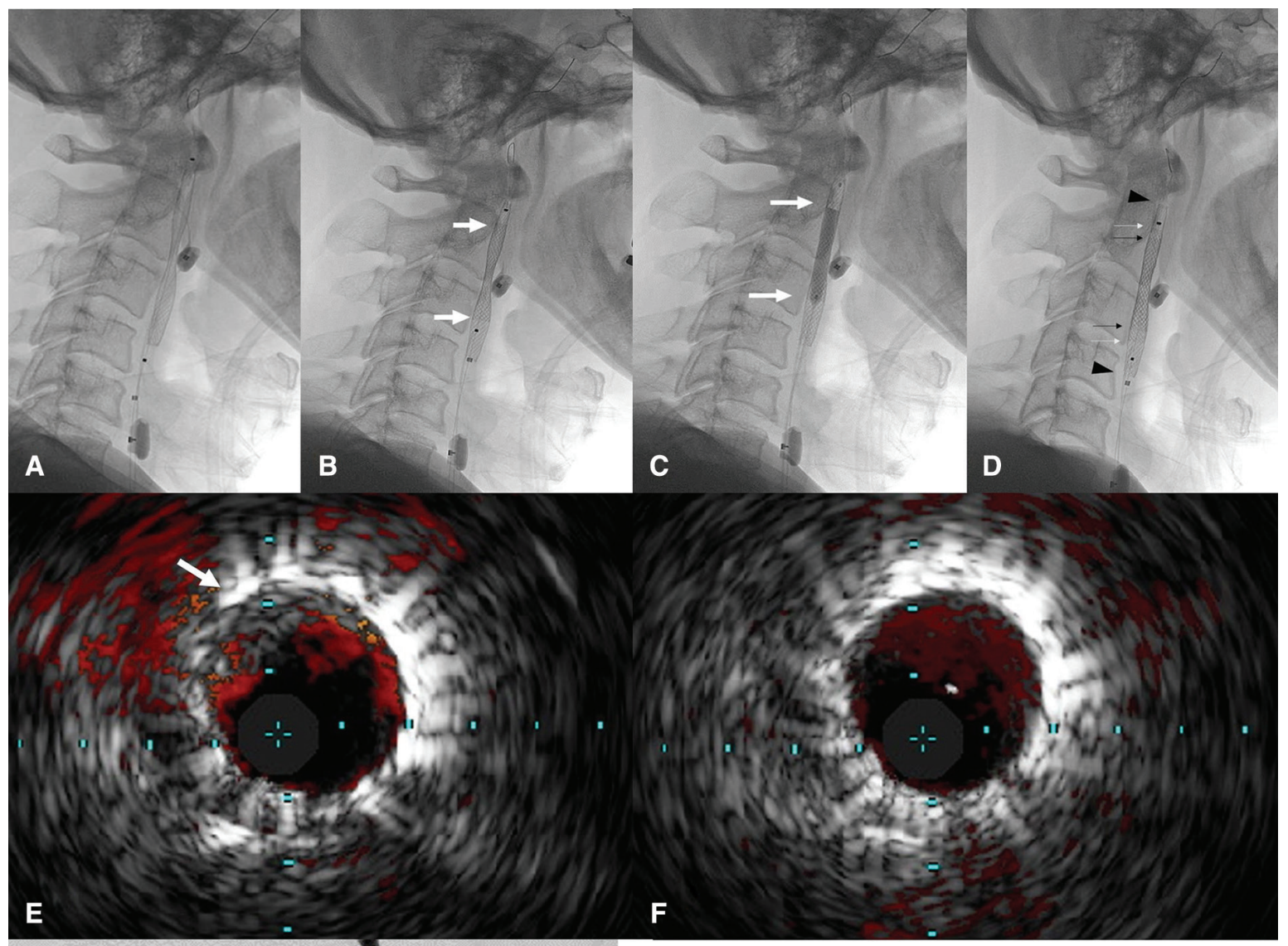

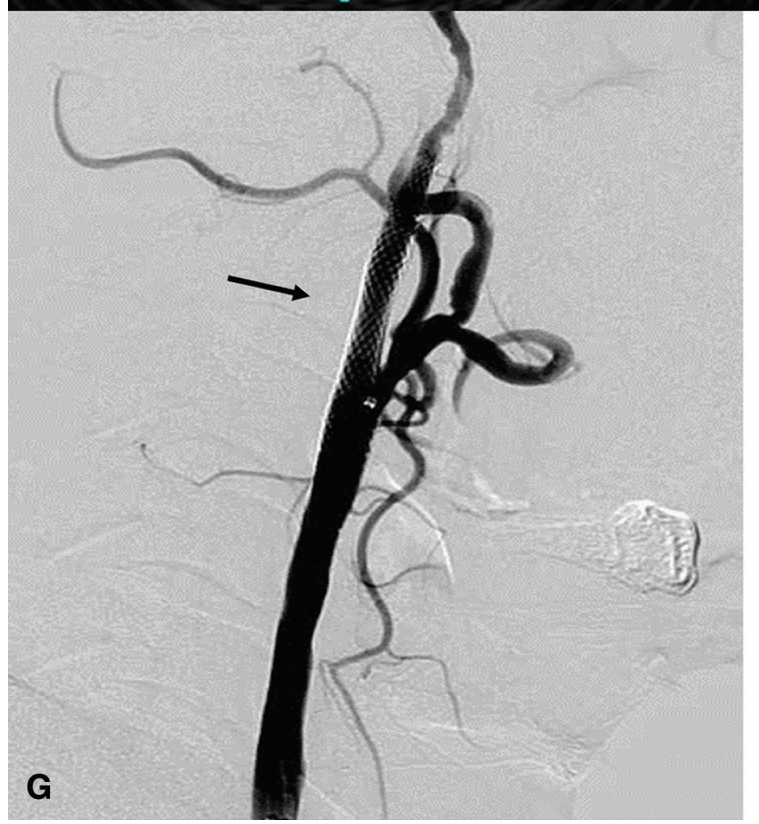

Fig. 3 CAS using a closed-cell stent-in-stent technique to treat $90 \%$ stenosis with unstable plaque at the origin of the right ICA. (A) Fluoroscopic image of the first stent placement (lateral view). An $8 \mathrm{~mm} \times$ $29 \mathrm{~mm}$ CWS was guided to cover the stenotic site, with proximal protection provided by a Mo.Ma Ultra device and distal protection by a FilterWire EZ. The first CWS was placed. (B) The second CWS $(6 \mathrm{~mm} \times 22 \mathrm{~mm})$ was guided inside the first stent. The second stent (arrows in B and C) was placed in a stent-in-stent manner. (C) Postdilation was performed using a $4 \mathrm{~mm} \times 40 \mathrm{~mm}$ balloon catheter. (D) The first (arrowheads), second (white arrows), and third stents (black arrows) were placed. (E) IVUS after placement of the second stent. The isoechoic area in the vascular lumen indicates intraluminal PP (arrow). (F) IVUS after placement of the third stent. Intravascular ultrasound was performed at the level of minimum lumen diameter confirming no PP. (G) Right common carotid angiography following additional third CAS (lateral view). Right common carotid angiography following CAS showed successful dilatation of the lesion without in-stent defect (arrow). CAS: carotid artery stenting; CWS: Carotid Wallstent; ICA: internal carotid artery; IVUS: intravascular ultrasonography; PP: plaque protrusion 
Taguchi $\mathrm{H}$, et al.

Hirata, Osaka, Japan). Subsequently, a Carotid Wallstent (CWS) $8.0 \mathrm{~mm} \times 29 \mathrm{~mm}$ (Boston Scientific) was inserted into the internal to common carotid arteries and another CWS ( $6 \mathrm{~mm} \times 22 \mathrm{~mm}$ ) was inserted using the stent-in-stent technique in accordance with the stenotic lesion. Postdilation was conservatively conducted using a $4.0 \mathrm{~mm} \times 40 \mathrm{~mm}$ Balloon catheter (Sterling; Boston Scientific). Intravascular ultrasonography (IVUS) (Volcano Visions PV 0.014P catheter with Chroma Flo; Volcano, Rancho Cordova, CA, USA) immediately postdilation showed PP, which was consistent with the area where the two stents overlapped. The third CWS $(6 \mathrm{~mm} \times 22 \mathrm{~mm})$ was additionally inserted to cover the PP area considering distal embolism and acute occlusion. IVUS and DSA immediately after insertion confirmed the disappearance of PP. There was no neurological symptom. No PP was noted on angiography after $10 \mathrm{~min}$, and the procedure was completed.

\section{Postoperative course}

After CAS, there was no abnormal neurological finding. On diffusion-weighted MRI the day after CAS, there was no high signal intensity area. On the fifth postoperative day, it was difficult to evaluate PP using ultrasonography (US), and angiography was performed on the seventh postoperative day (Fig. 4). Slight in-stent narrowing was observed, suggesting PP. $100 \mathrm{mg}$ of cilostazol was additionally administered and followed up. On the 10th postoperative day, US revealed no progression of PP, and the patient was discharged on the 14th postoperative day. During follow-up, there was no stroke. Follow-up angiography 3 months later confirmed the disappearance of PP. There was no restenosis (Fig. 5). At this point, Cilostazol administration was discontinued and switched to dual antiplatelet therapy (DAPT) with $100 \mathrm{mg}$ of aspirin and $75 \mathrm{mg}$ of clopidogrel.

\section{Discussion}

The purpose of CAS is to prevent cerebral infarction. Therefore, cerebral infarction as a perioperative complication must be particularly avoided if possible. However, its incidence reportedly ranges from $2.5 \%$ to $6.0 \%,{ }^{1,2)}$ and the incidence of perioperative cerebral infarction is slightly higher than that after CEA. ${ }^{1,2}$ Previous studies reported many risk factors for perioperative cerebral infarction related to CAS. In 2017, Kotsugi et al. ${ }^{4)}$ reported PP as a new risk factor. They noted PP in $2.6 \%$ of 328 patients who underwent CAS and found that it was strongly associated with perioperative cerebral infarction, whereas protection devices were not associated. Furthermore, they emphasized unstable plaque and open-cell stent use as predictive factors for PP. Considering the pathogenesis of PP, they hypothesized that stent use with a strong radial force and postdilation leads to the destruction of the fibrous capsule, resulting in protrusion of soft plaque through a stent. In the present case, unstable plaque and marked long-area stenosis were present, corresponding to a high risk for PP.

Considering the pathogenesis of PP, fine-mesh stents may be appropriate for the prevention of PP. Micromesh stents, which have finer mesh than standard stents, are applied in clinical practice. A meta-analysis ${ }^{6)}$ of four studies regarding micromesh stents showed that stent insertion was successful in all 556 patients, the incidence of perioperative stroke was $1.08 \%(n=6)$, and the severity of stroke was mild in these patients. The incidence of perioperative stroke is markedly lower than that in patients treated using conventional stents, which may have decreased through the inhibitory effects on PP. A recent multicenter prospective cooperative study $^{7}$ of a CASPER micromesh stent (Terumo, Tokyo, Japan) in Japan also reported an incidence of perioperative cerebral infarction of $1.4 \%$, being lower than that in patients treated using standard stents. In the future, the use of micromesh stents may improve treatment results in clinical practice.

As a method to prevent PP using standard carotid stents, Myouchin et al. ${ }^{5}$ proposed the stent-in-stent technique with closed-cell stents and noted no PP or perioperative cerebral infarction in all 35 patients. Furthermore, they emphasized that, when adopting this technique, the stent cell size was theoretically similar to or smaller than that of a micromesh stent. However, based on optical frequency domain imaging analysis, PP occurred even when a micromesh stent was used. Yamada et al. ${ }^{8)}$ reported the development of PP in 44\% of patients treated using CASPER stents. According to Umemoto et al., ${ }^{9)}$ PP was noted in $10.8 \%$ of those treated using C-guard stents and in $20.7 \%$ of those treated using Road Saver stents. Even when a micromesh stent is used, it may be impossible to completely prevent PP. The present case suggests that PP cannot be completely prevented even when adopting the stent-in-stent technique, as described for micromesh stents.

The optimal treatment for PP following CAS has not been established. Due to the limitation of additional stent insertion for PP after the stent-in-stent technique, the metal 


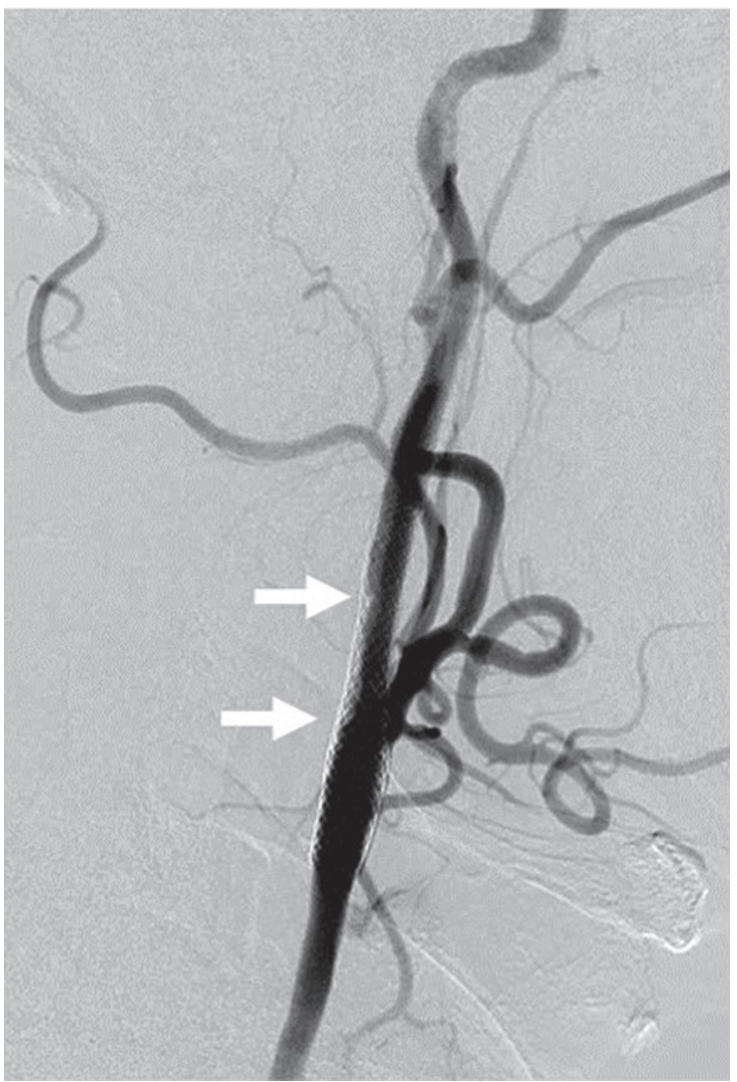

Fig. 4 Right common carotid angiography 1 week after CAS (lateral view). Lateral angiographic view at 1 week after CAS showing PP (arrow). CAS: carotid artery stenting; PP: plaque protrusion

volume is larger than that of a standard stent, and the incidences of restenosis and occlusion may increase. Therefore, long-term DAPT may be necessary to prevent restenosis or occlusion. Broussalis et al. ${ }^{10)}$ recommended that DAPT be continued for $\geq 6$ months when adopting a micromesh stent. Regarding the prevention of restenosis after CAS, Takayama et al. ${ }^{11)}$ reported that cilostazol significantly prevented restenosis in patients treated by CAS using CWS. Hashimura et al. ${ }^{12)}$ also suggested the usefulness of additional cilostazol administration in patients with PP within 1 week after CAS. Based on this, in the present case, $100 \mathrm{mg}$ of cilostazol was administered in addition to DAPT when angiography 7 days after CAS revealed PP. As angiography after 3 months confirmed the disappearance of PP and absence of restenosis, cilostazol administration was discontinued. Additional cilostazol administration may be considered as a treatment option for PP after CAS. Considering the treatment methods in the presence of PP, Kotsugi et al. $\left.{ }^{4}\right)$ recommended that a stent can be additionally inserted until the disappearance of PP when the shape of PP is evaluated as convex, and that follow-up can be

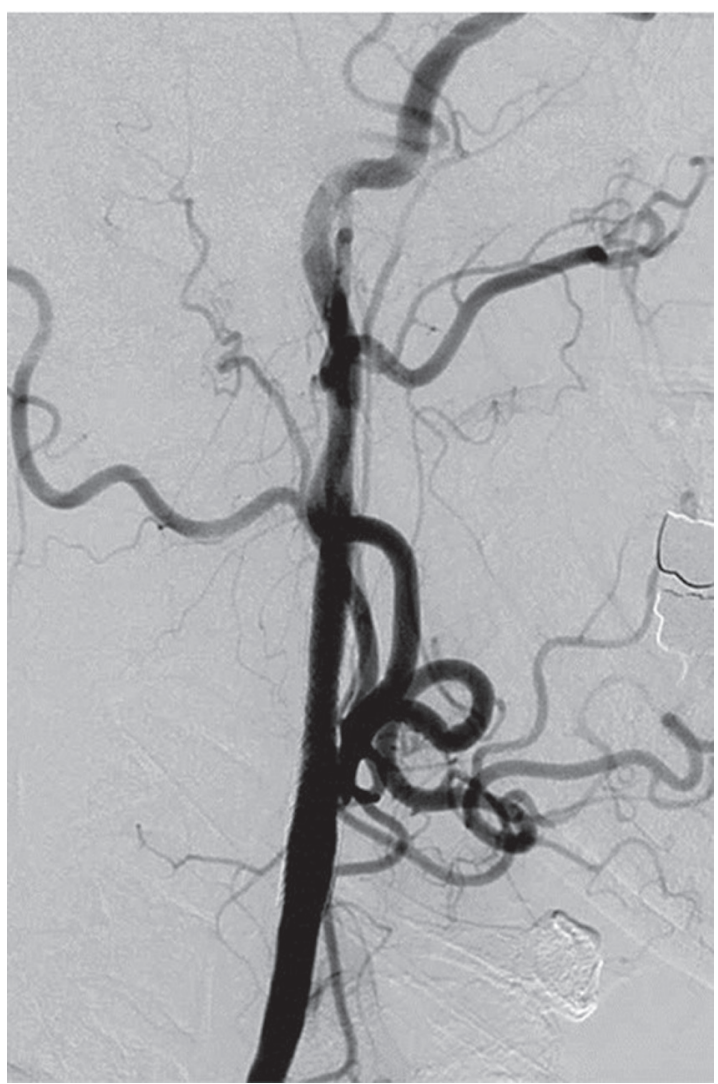

Fig. 5 Right common carotid angiography 3 months after CAS (lateral view). Lateral angiographic view at 3 months after CAS does not show PP. CAS: carotid artery stenting; PP: plaque protrusion

conducted for 5-10 $\mathrm{min}$ in patients with non-convex-type PP, followed by careful clinical follow-up within 30 days after CAS.

In the present case, the shape of PP was evaluated as convex and a CWS was additionally inserted. After confirming the disappearance of PP, the procedure was completed and there was no postoperative ischemic complication. However, DSA after 7 days revealed PP again. As PP may recur despite its disappearance during procedure, careful follow-up may be necessary within 1 week after CAS in highrisk patients for PP with unstable plaque, a long lesion, and large plaque volume such as the present case. Several investigators reported the usefulness of IVUS for PP diagnosis. The incidence of PP on IVUS during CAS ranges from 7.8 to $10 \% .{ }^{13,14)}$ Kotsugi et al. ${ }^{4)}$ reported that PP was detectable using IVUS in $27(7.6 \%)$ of 352 patients, and that DSA revealed PP in nine (2.6\%) of these. Additionally, they reported that there were no cases that could not be detected by IVUS but were detected by DSA, suggesting the importance of IVUS in the diagnosis of PP. In the present case, a diagnosis of PP was made using IVUS before eliminating 
Taguchi $\mathrm{H}$, et al.

protection, and we again recognized the importance of PP diagnosis using IVUS postdilation.

\section{Conclusion}

Even when CAS for carotid artery stenosis with unstable plaque is performed using the stent-in-stent technique, PP may occur. The importance of PP diagnosis using IVUS was suggested.

\section{Disclosure Statement}

The authors declare no conflict of interest.

\section{References}

1) Brott TG, Hobson RW, Howard G, et al: Stenting versus endarterectomy for treatment of carotid-artery stenosis. $N$ Engl J Med 2010; 363: 11-23.

2) Rosenfield K, Matsumura JS, Chaturvedi S, et al: Randomized trial of stent versus surgery for asymptomatic carotid stenosis. N Engl J Med 2016; 374: 1011-1020.

3) Takayama K, Taki W, Toma N, et al: Effect of pitavastatin on preventing ischemic complications with carotid artery stenting: a multicenter prospective study--EPOCH-CAS study. Cardiovasc Intervent Radiol 2014; 37: 1436-1443.

4) Kotsugi M, Takayama K, Myouchin K, et al: Carotid artery stenting: investigation of plaque protrusion incidence and prognosis. JACC Cardiovasc Interv 2017; 10: 824-831.

5) Myouchin $\mathrm{K}$, Takayama $\mathrm{K}$, Wada $\mathrm{T}$, et al: Carotid artery stenting using a closed-cell stent-in-stent technique for unstable plaque. J Endovasc Ther 2019; 26: 565-571.

6) Stabile E, de Donato G, Musialek P, et al: Use of duallayered stents in endovascular treatment of extracranial stenosis of the internal carotid artery: results of a patientbased meta-analysis of 4 clinical studies. JACC Cardiovasc Interv 2018; 11: 2405-2411.

7) Imamura $\mathrm{H}$, Sakai $\mathrm{N}$, Matsumoto $\mathrm{Y}$, et al: Clinical trial of carotid artery stenting using dual-layer CASPER stent for carotid endarterectomy in patients at high and normal risk in the Japanese population. J Neuro Intervent Surg 2020 Sep 15. doi: 10.1136/neurintsurg-2020-016250. [Epub ahead of print]

8) Yamada K, Yoshimura S, Miura $M$, et al: Potential of new-generation double-layer micromesh stent for carotid artery stenting in patients with unstable plaque: a preliminary result using OFDI analysis. World Neurosurg 2017; 105: 321-326.

9) Umemoto $T$, de Donato G, Pacchioni A, et al: Optical coherence tomography assessment of newgeneration meshcovered stents after carotid stenting. EuroIntervention 2017; 13: 1347-1354.

10) Broussalis E, Griessenauer C, Mutzenbach S, et al: Reduction of cerebral DWI lesion burden after carotid artery stenting using the CASPER stent system. J Neurointerv Surg 2019; 11: 62-67.

11) Takayama K, Taoka $T$, Nakagawa $H$, et al: Effect of cilostazol in preventing restenosis after carotid artery stenting using the carotid wallstent: a multicenter retrospective study. AJNR Am J Neuroradiol 2013; 33: 2167-2170.

12) Hashimura N, Mutoh $T$, Matsuda $K$, et al: Evaluation and management of plaque protrusion or thrombus following carotid artery stenting. Neurol Med Chir 2015; 55: 149-154.

13) Chiocchi M, Morosetti D, Chiaravalloti A, et al: Intravascular ultrasound assisted carotid artery stenting: randomized controlled trial. Preliminary results on 60 patients. J Cardiovasc Med 2019; 20: 248-252.

14) Shinozaki N, Ogata N, Ikari Y, et al: Plaque protrusion detected by intravascular ultrasound during carotid artery stenting. J Stroke Cerebrovasc 2014; 23: 2622-2625. 